\title{
REPRESENTAÇÕES DE SUPERVISÃO NA PERSPECTIVA DOS ENFERMEIROS COORDENADORES DE UM HOSPITAL DE ENSINO
}

\author{
NURSING SUPERVISION FROM THE PERSPECTIVE OF \\ NURSE COORDINATORS OF A TEACHING HOSPITAL
}

\section{REPRESENTACIONES DE SUPERVISIÓN EN LA PERSPECTIVA DE ENFERMEROS COORDINADORES DE UN HOSPITAL DE ENSEÑANZA}

\author{
Claudia Aparecida Dias ${ }^{1}$ \\ Dilma Costa Santos ${ }^{2}$ \\ Luciana de Oliveira Matias ${ }^{2}$ \\ Maria Lucia Silva Servo ${ }^{3}$ \\ Carmem Lúcia Albuquerque de Santana ${ }^{4}$ \\ Luiza Hiromi Tanaka
}

\begin{abstract}
Como citar este artigo: Dias CA, Santos DC, Matias LO, Servo MLS, Santana CLA, Tanaka LH. Representações de supervisão na perspectiva dos enfermeiros coordenadores de um hospital de ensino. Rev baiana enferm. 2018;32:e27422.

Objetivo: compreender a percepção dos Coordenadores de Áreas Assistenciais de Enfermagem sobre a supervisão de enfermagem realizada pelos enfermeiros assistenciais no contexto hospitalar. Método: estudo de abordagem qualitativa, fundamentado na Teoria das Representações Sociais. Foram entrevistados 16 coordenadores de enfermagem de um hospital de ensino. Os dados foram analisados pelo método Conteúdo Qualitativo-Temático. Emergiram três categorias empíricas: Supervisão de Enfermagem: Ferramenta Gerencial que Qualifica o Cuidado, Imbricamento entre Administrar e Cuidar e Supervisionar: Reflexo do Processo Formativo e Contexto Institucional. Resultados: a significação dada pelo grupo social dos coordenadores sobre a supervisão de enfermagem praticada revela uma prática gerencial de controle e pouco educativa, porém com impacto positivo para a qualidade assistencial. Conclusão: a percepção dos Coordenadores de Áreas Assistenciais de Enfermagem sobre a supervisão de enfermagem revelou deficiências na implementação atribuídas às lacunas da formação, às demandas excessivas no trabalho e à falta de capacitação em relação à prática da supervisão.
\end{abstract}

Descritores: Enfermagem. Teoria das Representações Sociais. Supervisão de Enfermagem.

Objective: to report the opinions of nurse coordinators on supervisory activities performed by nursing assistants in the hospital setting. Method: a qualitative study based on the theory of social representations. Sixteen nursing coordinators from a teaching hospital were interviewed. The data were analyzed using qualitative thematic content analysis.

\footnotetext{
Enfermeira. Doutora em Enfermagem. São Paulo, SP, Brasil.

Enfermeira. Mestre em Ciências. São Paulo, SP, Brasil.

Enfermeira. Doutora em Enfermagem. Professor Pleno da Universidade Estadual de Feira de Santana. Feira de Santana, Bahia, Brasil.

Médica. Doutora em Ciências em Saúde. Professora Afiliada do Departamento de Administração e Saúde Coletiva da Escola Paulista de Enfermagem, Universidade Federal de São Paulo. São Paulo, SP, Brasil.

5 Enfermeira. Doutora em Enfermagem. Professora Orientadora do Programa de Pós-Graduação da Escola Paulista de Enfermagem da Universidade Federal de São Paulo. São Paulo, SP, Brasil. luiza.hiromil @gmail.com
} 
Nursing supervision was divided into three categories: 1. a management tool that qualifies care, 2. an activity that interconnects nursing management and health care practice, and 3. a reflection of the formative process and the institutional context. Results: the opinions of nursing coordinators indicate that nursing supervision is a managerial practice based primarily on the exercise of authority but not on supervision training. However, this practice has a positive impact on the quality of care. Conclusion: the opinions of nursing coordinators about nursing supervision revealed deficiencies related to educational gaps, excessive work demands, and lack of supervision training.

Keywords: Nursing. Theory of Social Representations. Nursing Supervision.

Objetivo: comprender la percepción de Coordinadores de Áreas Asistenciales de Enfermería sobre supervisión de enfermería por enfermeros asistenciales en el contexto hospitalario. Método: estudio cualitativo, basado en la Teoría de las Representaciones Sociales. Se entrevistaron a 16 coordinadores de enfermería de hospital de enseñanza. Datos analizados por el método Contenido Cualitativo-Temático. Emergieron tres categorías empiricas: Supervisión de Enfermería: Herramienta Gerencial que Qualifica la Atención, Imbricamiento entre Administrar y Cuidar y Supervisionar: Reflejo del Proceso Formativo y Contexto Institucional. Resultados: la significación del grupo social de coordinadores sobre supervisión de enfermería practicada revela práctica gerencial de control y poco educativa, pero con impacto positivo para calidad asistencial. Conclusión: la percepción de Coordinadores de Áreas Asistenciales de Enfermería sobre supervisión de enfermería reveló deficiencias en la implementación atribuidas a las lagunas de la formación, a las demandas excesivas en el trabajo y falta de capacitación en relación a la práctica de supervisión.

Descriptores: Enfermería. Teoría de las Representaciones Sociales. Supervisión de Enfermería.

\section{Introdução}

A supervisão de enfermagem é um instrumento gerencial relacionado ao processo de trabalho do enfermeiro. Possibilita qualificar a sistematização da assistência de enfermagem em todos os níveis de atenção a saúde e fundamenta-se no planejamento, implementação e avaliação do trabalho da equipe de enfermagem, para qualificar o cuidado profissional ao paciente e família ${ }^{(1)}$.

Poucas foram as produções nacionais sobre o tema entre os anos de 1990 e 2010. Neste período, os textos versavam sobre a supervisão como função administrativa do enfermeiro ou destacavam o desenvolvimento de competências no processo de formação do enfermeiro para o exercício do cargo gerencial; outro aspecto também abordado nesse período foram as relações existentes entre a supervisão de enfermagem e as teorias administrativas que fundamentavam o processo de trabalho gerencial em enfermagem ${ }^{(2-3)}$. Na atualidade, as produções sobre o tema têm caminhado para a perspectiva da supervisão social ${ }^{(3)}$. Apesar do número insuficiente de produções sobre o tema, é possível observar uma dicotomia nas abordagens, que englobam a orientação de estudos de supervisão em enfermagem direcionados para a perspectiva do cuidado clínico e a outra voltada para a função gerencial, não levando em conta as interfaces do processo de trabalho em enfermagem assistir e gerenciar de forma indissociada ${ }^{(4-5)}$.

$\mathrm{Na}$ literatura internacional, a temática também é apresentada na perspectiva da prática assistencial e de gestão em enfermagem. Em países norte-americanos, as publicações científicas enfatizam a supervisão de enfermagem enquanto instrumento para a prática clínica, isto é, como modelo de avaliação para garantir o cuidado de enfermagem sistematizado ${ }^{(6-8)}$. Em países europeus, além do entendimento associado à prática clínica, também aparecem estudos relacionados à sua utilização na administração em enfermagem, no qual é compreendida como um possível diferencial qualitativo para a atuação na gestão de serviços de enfermagem ${ }^{(9)}$.

$\mathrm{Na}$ contemporaneidade, no contexto da gestão em enfermagem brasileira, após os adventos da política para a qualidade dos serviços prestados em saúde e da busca pela segurança do paciente, essa passa a ser compreendida como uma ferramenta administrativa capaz de propiciar processos horizontais no trabalho dos 
profissionais de enfermagem nas instituições de saúde, tanto em relação à gestão do cuidado como em relação à gestão de recursos humanos e recursos materiais ${ }^{(3,10)}$. Isso acontece porque essa ferramenta tem conseguido, no gerenciamento em enfermagem, associar a função gerencial de controle às ações de educação permanete, em uma perspectiva mais dialógica e participativa, deixando de ser utilizada pura e simplesmente como um instrumento de verificação de tarefas a serem cumpridas ${ }^{(3,10)}$.

A supervisão de enfermagem no século XXI modifica-se, adquirindo orientação processual ascendente, que representa de certo modo um caminhar objetivo intermediado por ações e reflexões contínuas a cada passo dado, o que coloca os atores que dela participam em constante reflexão-na-ação; daí, o fato de passar a ser caracterizada não mais como instrumento apenas, mas, agora, como método de intervenção no gerenciamento em enfermagem. Ao adquirir esse caráter, torna-se capaz de transformar tanto os processos como os agentes neles envolvidos ${ }^{(11)}$.

O conhecimento das transformações ocorridas em relação à prática da supervisão de enfermagem no Brasil e no mundo, e suas correlações com as políticas públicas de gestão do cuidado em enfermagem e saúde, levaram à reflexão mais profunda sobre o exercício da supervisão enquanto prática assistencial e gerencial de enfermagem nas instituições hospitalares, na atualidade. As leituras em busca de aprimoramento sobre o tema trouxeram inquietações e curiosidades importantes em relação à aplicação de uma das ferramentas gerenciais mais importantes no trabalho do enfermeiro. Justamente pelo seu poder tranformador da prática assistencial, tem-se perguntado se esta tem produzido ressonância no cotidiano da prática dos enfermeiros e na qualidade do cuidado prestado pela equipe de enfermagem.

O reconhecimento de que a avaliação desse fenômeno perpassa pela gestão do cuidado e do gerenciamento em enfermagem leva a buscar-se ampliar o olhar sobre o processo de trabalho do enfermeiro assistencial e de toda a dinâmica que envolve o cuidar e gerenciar em enfermagem e saúde, em uma instituição de assistência à saúde, nos moldes da assistência hospitalar. Daí, a opção por conhecer o fenômeno pela compreensão introjetada de supervisores gerais de enfermagem, grupo social representativo do ser e fazer enfermagem, na perspectiva desejada.

A constatação de que, nos últimos 20 anos, apesar das mudanças curriculares para o ensino de enfermagem e das discussões sobre as concepções e valores de gestão, a supervisão de enfermagem ainda pode contemplar resquícios do modelo tradicional de gestão, isto é, apresentar caráter de controle e assentar-se em experiências gerenciais empíricas e pouco participativas, permitiu a elaboração do seguinte questionamento: Qual é a representação atual de supervisão em enfermagem no contexto hospitalar percebida pelo grupo social dos Coordenadores de Áreas Assistenciais de Enfermagem (CAAE)?

Para desvelar esse fenômeno, este estudo tem como objetivo compreender a percepção dos Coordenadores de Áreas Assistenciais de Enfermagem sobre a supervisão de enfermagem realizada pelos enfermeiros assistenciais no contexto hospitalar.

\section{Método}

Trata-se de um estudo exploratório de abordagem qualitativa, que adota como referencial teórico-metodológico a Teoria das Representações Sociais (TRS), possibilitando a apreensão dos sentidos, significados e percepções de um determinado fenômeno social, construídos através das relações estabelecidas em um determinado grupo social e expressadas por sua linguagem.

As representações sociais acontecem por intermédio de dois processos: a objetivação e a ancoragem. A objetivação transforma algo abstrato, objeto ou fenômeno, não familiar em algo concreto, retirando-o do imaginário, tornando-o conhecido por meio da linguagem. A ancoragem é um processo que possibilita classificar, avaliar ou codificar algo, de forma a "amarrar" representações de memória que são estranhas, 
colocando-as em um contexto familiar, transformando-as em algo conhecido e compartilhado ${ }^{(12)}$.

No contexto social de enfermagem, a todo momento, são construídas representações sociais sobre a prática da supervisão que, por meio desta investigação, poderão deixar o universo imaginário dos participantes e emergir em seus discursos, revelando as representações da supervisão realizada pelos enfermeiros no cotidiano assistencial.

O cenário de estudo foi o Hospital da Universidade Federal de São Paulo (UNIFESP), no município de São Paulo. Foram entrevistados 16 enfermeiros no cargo de CAAE. Essa escolha deu-se porque esses colaboradores poderiam conhecer em profundidade o fenômeno estudado e por serem responsáveis pela avaliação da prática de enfermeiros assistenciais. Deveriam possuir, no mínimo, 2 anos de experiência no cargo e estarem presentes no período da coleta de dados. Estes critérios foram responsáveis pela exclusão de 7 participantes do rol de 23 colaboradores, número obtido junto à Diretoria de Enfermagem. Assim, 6 colaboradores foram excluídos, por não atenderem aos critérios de seleção e 1 por ter participado do teste piloto, para adequação do roteiro de entrevista.

A aproximação com os entrevistados deu-se por contato pessoal nos setores de sua atuação, momento em que foram esclarecidos em relação aos objetivos propostos e feito o agendamento das entrevistas. Estas foram individuais, realizadas pelo pesquisador após a assinatura do Termo de Consentimento Livre e Esclarecido (TCLE) e duraram em média 35 minutos. A pergunta norteadora foi: Como você percebe a supervisão de enfermagem realizada pelo enfermeiro no cotidiano do trabalho? Depois de cada entrevista foi realizada a sua transcrição. Nesse período, obteve-se um corpus de análise, representado por um documento de 141 páginas. Depois das transcrições, cada entrevista foi encaminhada a seu autor, por e-mail, para validação de seu conteúdo.

Os resultados das entrevistas foram analisados pela técnica de Análise de Conteúdo de Dados Qualitativos ${ }^{(13)}$, que tem como fim gerar teorias, hipóteses ou temas baseados nos referenciais teóricos adotados. Em síntese percorreu-se o seguinte caminho: preparo dos dados, leitura abrangente para limpar ruídos; imersão e leitura reflexiva do corpus de análise para revisão dos resultados; codificação de primeiro nível para formação de unidades de dados e significados; agrupamento de categorias por semelhança e conformidade com o objeto de estudo para compreensão dos significados ${ }^{(13)}$.

Em todas as etapas desta pesquisa, foram respeitados os aspectos éticos recomendados pela Resolução n. 510, de 2016, do Conselho Nacional de Saúde (CNS) ${ }^{(14)}$. A pesquisa foi autorizada pelo Comitê de Ética em Pesquisa (CEP) da UNIFESP/Hospital São Paulo, com o Parecer n. 503.577, aprovado em 20/12/2013. Foi também aprovada pela Coordenadoria de Ensino e Pesquisa do Hospital Universitário, sob Protocolo n. 70 , de 10 de outubro de 2013.

\section{Resultados}

Os agrupamentos das categorias deram origem aos temas que possibilitaram a compreensão das Representações Sociais (RS) sobre a supervisão de enfermagem realizada pelo enfermeiro no contexto hospitalar na perspectiva do grupo social dos CAAE. As três categorias temáticas apreendidas foram: Supervisão de enfermagem: ferramenta gerencial que qualifica o cuidado; Supervisão de enfermagem: imbricamento entre administrar e cuidar; e Supervisão: reflexo do processo formativo e contexto institucional.

\section{Supervisão de Enfermagem: Ferramenta Gerencial que Qualifica o Cuidado}

Esta primeira categoria, foi intitulada devido à significação atribuída pelos participantes à supervisão enquanto instrumento gerencial para o cuidar. A supervisão foi compreendida pelo grupo social como uma ferramenta de gestão para o direcionamento da dinâmica de trabalho da equipe de enfermagem e a garantia do provimento das necessidades locais (recursos humanos, físicos e materiais) para a efetivação do 
cuidado de enfermagem. É o que mostra o fragmento de discurso, a seguir:

[...] aquilo que diz respeito à programação dos recursos físicos, materiais e humanos para a assistência, assim como a delegação das tarefas, de acordo com a competência técnica e as habilidades de cada um [...] É a supervisão de fato das tarefas executadas pelos profissionais, além dos resultados que se está obtendo e a avaliação em relação aos resultados esperados. (E14).

Considerando o princípio da ancoragem, observou-se que a representação social apreendida reconhecia a supervisão como um recurso gerencial para a organização do trabalho e a verificação da prática. A crença de que a supervisão destinava-se à verificação de técnicas e ao provimento de recursos era uma representação forte no grupo social dos coordenadores e apareceu sempre que estes descreveram a supervisão do enfermeiro assistencial.

Essas influências tradicionais representaram-se nos discursos principalmente no que se referia à organização de recursos humanos, materiais e à organização do ambiente assistencial. Os participantes reconheceram sua importância para o alcance dos objetivos da supervisão, isto é, ofereciam melhores condições de trabalho à equipe e proporcionavam qualidade ao cuidado de enfermagem:

A supervisão de enfermagem precisa ser feita tanto para a
organização do trabalho, porque dessa forma você prio-
riza, organiza e qualifica o trabalho para o paciente, o
cuidado fica qualificado específico para aquele paciente.
Então a supervisão é importantíssima. (E2).

Supervisão é você estar verificando o trabalho de enfermagem, direcionando a sua equipe, avaliando, supervisionando o trabalho em geral, a qualidade da assistência de enfermagem do setor. (E11).

Apesar do tecnicismo administrativo presente nas representações de supervisão, observou-se a compreensão dos coordenadores sobre sua finalidade, isto é, ser uma ferramenta que permite a qualificação da prática assistencial e gerencial do enfermeiro.

A RS a seguir revela que os enfermeiros realizavam a supervisão na forma de checagem ao acaso, verificando inconformidades, sem planejamento ou sistematização das ações:

[...] não é uma coisa programada. O enfermeiro não consegue ficar o tempo todo em cima do auxiliar [...] Não é feito assim: vamos boje lá fazer uma supervisão, não!
Porque é assim: o enfermeiro circula muito nos quartos, na enfermaria como um todo, na semi-intensiva. $E$ O momento que ele tem para bater o olho e ver o que está acontecendo de errado ou não. (E5).

Embora esteja presente nos discursos o compromisso com a qualidade da assistência, a supervisão no local de estudo era realizada pelos enfermeiros de acordo com as necessidades e situações pontuais de cada unidade. Os discursos não apontaram a aplicação de instrumentos para supervisão sistematizada, isto é, instrumentos fundamentados no planejamento, na execução e na avaliação das práticas da equipe de forma completa. Além da ausência do planejamento, poucas foram as representações sobre a função educativa da supervisão do enfermeiro:

Alguma falha ou erro que a gente vê que se torna repetido, elaboramos alguns treinamentos. Isso pode vir através dos residentes [...] O enfermeiro não fica treinando, porque ele fica muito sobrecarregado. (E6).

As RS apreendidas sinalizaram que a orientação educativa, quando realizada pelos enfermeiros, acontecia de forma individualizada $\mathrm{e}$ para redimir erros. Isto, entretanto, não deveria acontecer, pois a função educativa é um dos pilares de sustenção da supervisão enquanto prática de cooperação e corresponsabilização.

\section{Supervisão de Enfermagem: Imbricamento entre Administrar e Cuidar}

As RS da supervisão realizada pelos enfermeiros assistenciais para o grupo social dos CAAE apontaram para o imbricamento existente entre as ações administrativas rotineiras e $\mathrm{O}$ cuidar no cotidiano do trabalho.

Diante da diversidade das práticas dos enfermeiros no cotidiano de acordo com os CAAE na esfera do cuidar e administrar, elaborou-se um quadro organizando as representações das atividades ancoradas em três subdivisões cujo rol encontra-se disposto no Quadro 1: ações para garantir a orientação do trabalho em equipe, para organização do ambiente assistencial e Cuidado assistencial direto e indireto. 
Quadro 1 - Ações administrativas e instrumentos para supervisão do cuidado de enfermagem

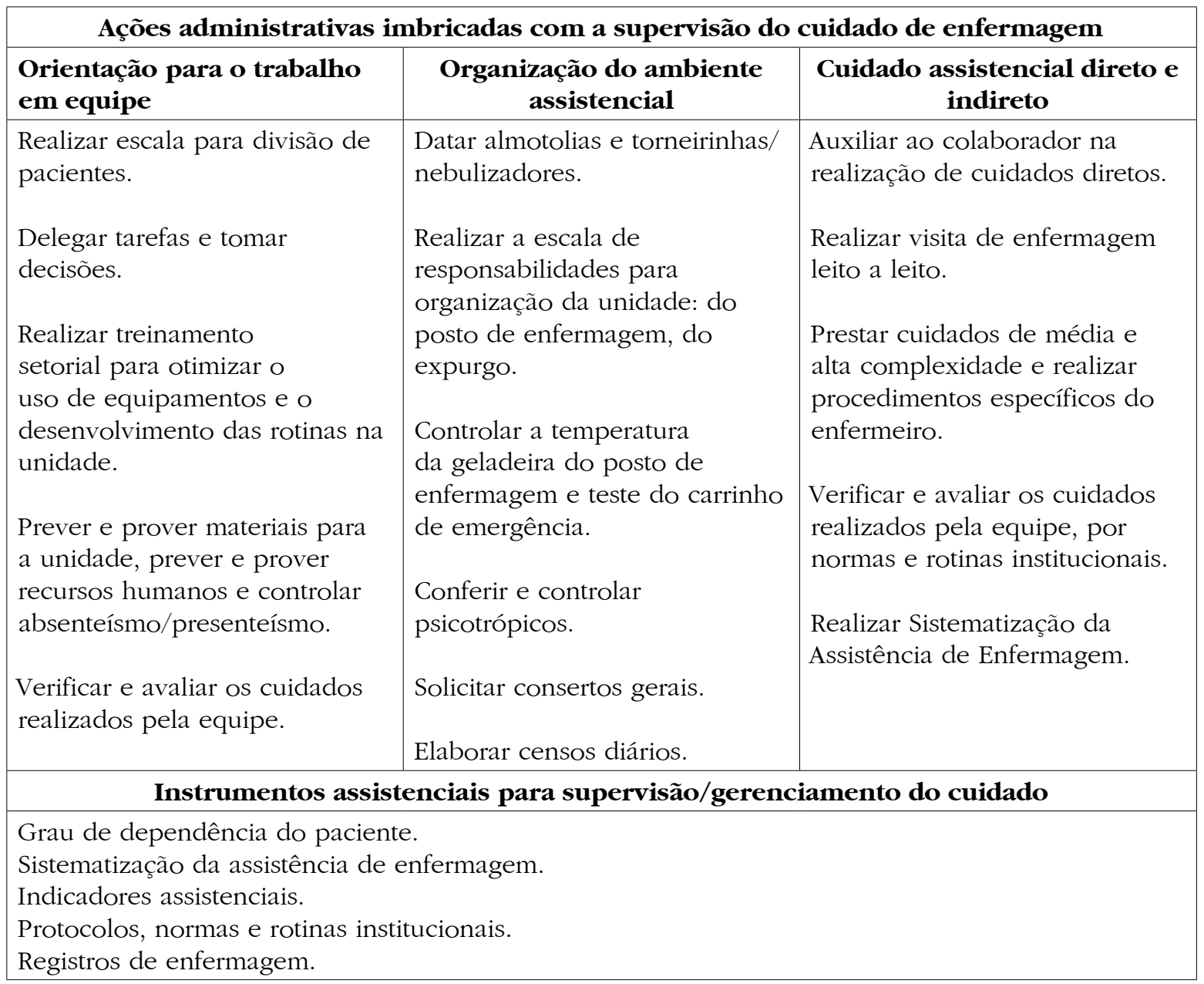

Fonte: Elaboração própria.

As representações sociais das atividades realizadas durante a supervisão pelos enfermeiros assistenciais sob a ótica dos CAAE, evidenciaram, de forma objetiva, que a supervisão realizada pelos enfermeiros contemplava a interrelação entre ações de gerenciamento do cuidado e ações de cuidado direto e indireto ao paciente. Desse modo, instrumentos administrativos, como a supervisão de enfermagem, são realmente necessários para realizar a implementação da assistência sistematizada em enfermagem.

Nos discursos, evidenciou-se também o predomínio de ações de controle da prática profissional, ancorando-se a ideia da utilização da supervisão de enfermagem como ferramenta de gestão do cuidado:

Ele faz toda a distribuição da escala. Ele consegue estar junto de seus colaboradores em procedimentos de complexidade. Ah, se vai dar um banho, então, o enfermei- ro entra junto, para fazer a avaliação do paciente [...] Consegue também ver, junto com o escriturário, a provisão de materiais e medicações da cota diária e também outras rotinas [...] Então, ele faz todo esse serviço, fora a SAE, que também é sua função. (E7).

Você olha a escala e fala: "Hoje está apertado". Então, boje eu tenho poucos funcionários para muitos pacientes. Como meus pacientes estão? [...] Então, eu visualizo o que é prioridade, passo para cada funcionário como tem que ser feito o processo de trabalbo. (E11).

Os CAAE, em seus discursos, demonstraram que os enfermeiros realizavam cuidados diretos e indiretos durante a supervisão de enfermagem. A ancoragem do gerenciar e cuidar na supervisão do enfermeiro assistencial era representada por um imbricamento desconhecido, isto é, os coordenadores, em sua maioria, não tinham consciência desse imbricamento, já que descreviam as diversas ações executadas durante a supervisão, sem expressar o reconhecimento de sua intersecção. 
Embora o imbricamento tenha sido uma representação significativa, em alguns discursos foi possível observar que os enfermeiros coordenadores ancoravam a crença de que o enfermeiro assistencial, ao realizar ações gerenciais, essencialmente burocráticas, distanciava-se do cuidado direto:

[...] temos uma estrutura que não nos permite fazer isso, pela característica do bospital. Principalmente nesses últimos seis meses, a gente está tendo muitos desgastes com faltas, falta de materiais imprescindiveis [...] porque, no momento que eu estaria ajudando os colaboradores a prestar uma melhor assistência, eu estou correndo atrás de uma coisa que não é minha função realmente. (E8).

[...] só que, muitas vezes, o enfermeiro deixa de fazer este papel. Para ele é muito mais fácil, ele só organizar, mandar: faz isso assim. É claro que isso é muito mais fácil; sensibilizar é o mais difícil. Eu vejo que é uma dificuldade de quase todos os enfermeiros [...] (E13).

Supervisionar: Reflexo do Processo Formativo e do Contexto Institucional

$\mathrm{Na}$ terceira e última categoria construída, os CAAE sinalizaram que a supervisão do enfermeiro estava aquém do desejado e associavam o fato às deficiências do processo formativo, do conhecimento e das habilidades próprias da profissão, sobretudo a gestão do cuidado, o que leva às consequências de não conseguirem efetuar a supervisão de enfermagem de forma qualificada. É o que se observa nas falas a seguir:

Acho que eles vêm muito despreparados da faculdade,
tanto da particular quanto da pública [...] Eu acho que
as universidades deveriam rever o currículo institucional
e estar preparando mais o enfermeiro. (E12).

Eles não conseguem ter esta noção de saber visualizar e saber quando está fazendo errado e ensinar o correto. Eles não sabem como fazer [...] não sei se a carga horária está mudando muito na escola, e eles não estão indo tanto para a prática [...] Eu vejo eles muito despreparados. (E16).

A sobrecarga das atribuições do enfermeiro em sua jornada de trabalho foi outro fator institucional representado pelos coordenadores como responsável pela pouca eficiência da supervisão realizada. O grupo social dos CAAE referiu que a escassez de recursos humanos e materiais na instituição sobrecarregava as funções do enfermeiro assistencial e atrapalhava a execução da supervisão de enfermagem.
Se fosse um pouquinho menos apertado, daria para o enfermeiro perguntar para sua equipe: o que vocês acham que está faltando para vocês? Estão com dúvidas de alguma coisa? [...] é tão pouco funcionário que o enfermeiro não consegue. Ele não tem tempo [...] ele poderia sentar mais com sua equipe [...] O grande problema deste hospital é a falta de funcionários e materiais. (E11).

Nas RS dos CAAE, outra questão que foi apontada como prejudicial à prática da supervisão foram às deficiências relativas ao dever institucional de prover educação no ambiente de trabalho. Entretanto, os coordenadores atribuíam também responsabilidade individual desses profissionais para com a sua formação, expressando que existia, de certa forma, um estado de apatia, pois os enfermeiros assistenciais buscavam pouco a educação continuada. Os discursos dos enfermeiros coordenadores ancoravam certa expectativa em relação à proatividade dos enfermeiros assistenciais para com a sua capacitação individual, mas reconheciam que a instituição deveria, de forma mais ativa, implementar ações educativas em relação à supervisão de enfermagem:

Eu acho que todos os enfermeiros deveriam ter um pro-
grama de educação permanente [...] Só que falta um
olhar da alta direção para treinamentos para os colabo-
radores, porque hoje, tirando o treinamento online, não
temos mais nenhum. (E8).
Sempre a gente tem que melhorar, estar se atualizando
e tem que passar isso para seu auxiliar e para o técnico
[...] Têm alguns enfermeiros que são mais conformados,
estão na zona de conforto, não tentam se atualizar e
não passam algumas atualizações para sua equipe. (E9).

A questão do despreparo para supervisionar e as poucas ações educativas institucionais para suprir essa deficiência é uma representação importante no discurso dos CAEE. Evidencia-se que o aprendizado para o desenvolvimento das competências de gestão relacionadas à supervisão de enfermagem, na maioria das vezes, dá-se no fazer cotidiano, nas relações interprofissionais. É o que mostram os fragmentos seguintes:

Ele vai adquirindo na raça [...] porque não tem uma instrução, alguém com outra visão para mostrar para ele. Então, ele vai aprendendo. Eles que constroem. (E15).

Tem que amadurecer e nada melhor que o dia a dia para esse amadurecimento e nós, mais velhos, dando o suporte para eles. Bateu de frente? Opa, agora vamos apaziguar. Depois que está tudo certinho, a gente fala: "Olha, não faz assim, da próxima vez tente desse jeito.”. (E16). 


\section{Discussão}

No tocante à categoria "Supervisão de enfermagem: ferramenta gerencial que qualifica o cuidado", observa-se que a principal inferência refere-se à identificação introjetada da função controle pela representação da ação de verificação dos processos envolvidos no desenvolvimento do trabalho do enfermeiro com sua equipe. A verificação é considerada uma atividade da função controle no gerenciamento de enfermagem. Existe para garantir a identificação de problemas e possibilitar adequações das ações dos colaboradores aos padrões e normas estabelecidos pela instituição. O objetivo maior é garantir a manutenção da qualidade do trabalho executado. Isso acontece por meio de orientações técnicas e administrativas do enfermeiro a sua equipe ${ }^{(15)}$.

A supervisão de enfermagem tem seus pilares constitutivos nos fundamentos da teoria clássica da administração. Daí apresentar características como divisão do trabalho e hierarquização de funções. Apesar das transformações sociais, estudos demonstram que a prática da supervisão de enfermagem ainda apresenta características conservadoras do modelo de trabalho taylorista/fordista e da organização burocrática de Fayol $^{(3,10)}$.

$\mathrm{Na}$ atualidade, a supervisão de enfermagem, por meio do uso do planejamento situacional apresenta-se fundamentada no levantamento de problemas de forma mais contextualizada, o que possibilita envolver todos os profissionais da equipe de enfermagem na elaboração do programa assistencial do paciente ${ }^{(16)}$. A ausência do uso do planejamento enseja reflexão a respeito da necessidade do desenvolvimento de treinamentos em relação à prática da supervisão sistematizada, para que possa transformar-se em uma supervisão integralizadora ${ }^{(17-18)}$.

Em uma revisão integrativa realizada no Brasil, sobre a supervisão de enfermagem no âmbito hospitalar, identificou-se ainda que a maioria dos enfermeiros pratica a supervisão de enfermagem segundo o modelo tradicional, com caráter tecnicista, por meio de protocolos assistenciais normatizados para controlar as ações das equipes na produção do trabalho cotidiano. No entanto, evidenciam-se também experiências relacionadas à prática da supervisão baseada em participação coletiva e corresponsabilização entre o enfermeiro e sua equipe, de modo menos hierarquizada e mais educativa ${ }^{(2)}$.

Essa realidade em relação à supervisão de enfermagem também pode ser observada no trabalho gerencial do enfermeiro em unidades de saúde. Entretanto, apesar dos avanços e das mudanças de paradigma em relação ao processo de trabalho do enfermeiro e da equipe de enfermagem na atenção primária, ainda há muitos supervisores de enfermagem que implementam a supervisão na forma de controle, apenas com fins corretivos, pouco educativa, mesmo tratando-se de uma realidade na qual as relações de trabalho são mais flexíveis e horizontalizadas $^{(18-19)}$. Os achados da literatura especializada, tanto na realidade do trabalho na atenção primária e secundária como no contexto hospitalar, foram semelhantes ao que foi evidenciado no presente estudo. Neste, inclusive, não foi possível perceber correlação entre a supervisão de enfermagem e a função educativa, dada a ênfase presente nos discursos em relação à função controle.

As ações educativas na supervisão permitem aos profissionais a reflexão-na-ação. Quando isso não acontece, os profissionais atuam de forma mecanicista e descontextualizada, desfavorecendo a horizontalização na dinâmica do trabalho. A educação permanente e a supervisão compartilhada são as ferramentas administrativas que constroem e reconstroem o cotidiano da prática e também apresentam potencial significativo para superar o modelo assistencial biologicista, no qual poucos decidem como e o que fazer e aqueles que fazem, na maioria das vezes, não sabem por que fazem desse ou daquele modo. Portanto, é essencial modificar esse estilo de supervisão ${ }^{(17)}$.

A imersão nos resultados da categoria "Supervisão de enfermagem: imbricamento entre administrar e cuidar" possibilitou a elaboração de um constructo que representa a supervisão de 
enfermagem enquanto ferramenta de gestão que possibilita a interrelação entre cuidar e gerenciar o cuidado, sendo o enfermeiro considerado o profissional responsável pela efetivação dessa prática. Isso acontece porque, quando supervisiona, ele realiza o planejamento, a execução e a avaliação das demandas assistenciais que envolvem recursos humanos e materiais, tudo isso de forma a garantir a "convergência entre essas duas esferas" ${ }^{(19-20)}$.

As ações de cunho administrativo deveriam auxiliar na organização e na dinâmica do trabalho de enfermagem, proporcionando subsídios para a tomada de decisão gerencial e qualificação da assistência. Entretanto, estudos demonstram que a gestão burocrática tem afastado o enfermeiro da prática assistencial ${ }^{(16,19)}$.

Em um estudo sobre as implicações da supervisão de enfermagem nos resultados do trabalho em equipe e sua correlação com a qualidade do cuidado, os autores referem que "[...] o enfermeiro em seu trabalho possui uma série de responsabilidades e deveres para com a equipe de enfermagem, clientela e instituição em que atua e para isso ele se organiza através dos processos de trabalho de assistir, investigar, gerenciar e educar" ${ }^{\text {(21:15) }}$. Assim, para que se alcance a qualidade e a segurança do cuidado é imprescindível a integração entre o cuidar e o gerenciar, e a supervisão de enfermagem é a chave para essa mudança assistencial ${ }^{(21)}$.

A última categoria presente na expressão dos resultados obtidos trata das lacunas existentes no mundo do trabalho e sua interface com a formação, no que se refere ao desenvolvimento de conhecimentos e competências próprias para realizar a supervisão de enfermagem. Em relação aos conhecimentos sobre gerenciamento em enfermagem na graduação, têm sido frequentes as sinalizações sobre deficiências e lacunas no tocante ao desenvolvimento de competências gerenciais, de modo geral ${ }^{(22)}$. Outro ponto a discutir sobre esta questão é o fato de que as instituições de saúde têm investido pouco no desenvolvimento de conhecimentos e habilidades específicos para a supervisão de enfermagem e gestão do cuidado, o que faz com que os enfermeiros busquem capacitações na forma de educação continuada ${ }^{(22)}$.

Estudos sobre o processo de trabalho atribuem a baixa eficiência da supervisão ao fato de os enfermeiros executarem várias atividades que, muitas vezes, não fazem parte de seu papel profissional ${ }^{(0,23)}$. A sobrecarga teria resultado negativo para a execução da supervisão de enfermagem, podendo torná-la superficial e pouco contextualizada em relação às demandas dos profissionais de enfermagem envolvidos na assistência ${ }^{(21-22)}$. A falta de eficiência para supervisionar também é associada à falta de conhecimentos específicos sobre esta ação gerencial. Esses conhecimentos, quando deficientes em relação à bagagem trazida da graduação, deveriam ser desenvolvidos adequadamente no início da vida profissional. Entretanto, os coordenadores e as instituições que recebem egressos não têm conseguido suprir tal deficiência ${ }^{(22,24)}$.

É preciso reconhecer que a falta de conhecimentos e de habilidades para supervisionar, além da formação também perpassa pela deficiência de aprendizagem prática, pois, na maioria das instituições, essa aprendizagem ainda é realizada de forma empírica por meio da proatividade de enfermeiros dos campos de prática, que repassam suas vivências aos iniciantes. Entretanto, quando o fazem, na maioria das vezes não observam a utilização dos fundamentos do gerenciamento em enfermagem nessa ação educativa ${ }^{(23-25)}$.

Os CAAE, como enfermeiros responsáveis também pela supervisão de enfermagem dos supervisores assistenciais, devem corresponsabilizar-se pelo aprendizado e exercício da supervisão, pois desenvolver competências para supervisionar é um processo contínuo de aprendizagem que se dá na prática e na intersubjetividade do trabalho cotidiano de enfermagem ${ }^{(24-25)}$.

Os resultados apreendidos pela objetivação e ancoragem remetem ao entendimento de que a supervisão de enfermagem realizada pelos enfermeiros assistenciais é uma atividade própria do ser e do fazer do enfermeiro. Este inicia sua construção antes de inserir-se no mundo da prática, mas só projeta-se e introjeta-se como atitude profissional qualificada quando este, ao longo 
de sua vivência, agrega conhecimentos e experiências das relações interprofissionais e dos valores institucionais ${ }^{(20)}$. Os resultados observados no local de estudo corroboram essa argumentação, porque, também no contexto institucional dos participantes, a supervisão de enfermagem sinaliza a necessidade do desenvolvimento de políticas institucionais de aproximação com o modelo da supervisão social, com ênfase para a educação permanente reflexiva.

A realização deste estudo tem como limitação referir-se apenas a um lócus determinado. Entretanto, a sua realização em outras realidades é possível e recomendada, pois poderá trazer novas perspectivas de interpretação sobre o objeto investigado, que podem ir além daquilo que foi percebido pelos pesquisadores.

O estudo pode contribuir para a reflexão sobre a atividade do enfermeiro assistencial na esfera da supervisão em enfermagem, prática imprescindível no cotidiano de trabalho em enfermagem e que deve ser compreendida e implementada como método de intervenção no processo gerencial em enfermagem. Outra colaboração diz respeito à ampliação da discussão sobre a flexibilização da supervisão em enfermagem enquanto expressão do modelo de gestão participativa, temática com significado tanto para profissionais inseridos na prática hospitalar (coordenadores, supervisores, gestores) como docentes que atuam no ensino de graduação em enfermagem, para o fortalecimento da gestão do cuidado, pois, sem a supervisão, não há como qualificar a assistência em enfermagem. À luz das percepções apreendidas em relação à prática da supervisão em enfermagem, sugere-se estudos de intervenção com a utilização de instrumentos sistematizados para colaborar com o processo de mudança paradigmática em curso.

\section{Conclusão}

As RS dos CAAE sobre a supervisão de enfermagem e sua aplicação prática no cotidiano da assistência de enfermagem foi a expressão de princípios e valores relativos à supervisão tradicional, em que os enfermeiros assistenciais orientam suas ações pelo saber positivista e pelas teorias clássicas da administração.

Ancora-se, como constructo principal, o controle. Este embasa programas educativos relacionados a erros e inconformidades do cotidiano, no qual a supervisão é representada como um instrumento de gestão que permite o alcance da qualidade assistencial e a contenção de custos. As ações educativas acontecem de forma pontual e descontextualizadas, com poucas representações de participação do enfermeiro enquanto agente que educa para a reflexão-na-ação. O grupo ancora a angústia do despreparo para supervisionar como resultado de deficiências e lacunas relativas ao processo de formação, além de fatores institucionais de divisão do trabalho e educação para a supervisão, o que se traduz na falta de competência gerencial para o exercício da supervisão de enfermagem social.

O cuidado e a organização da unidade são considerados pelo grupo social as principais ações relacionadas à supervisão de enfermagem. No local de estudo, a sua execução é rotineira, porém sem planejamento e sistematização e com pouca participação da equipe. É, portanto, praticada, mas ainda não se configura a incorporação das mudanças paradigmáticas emergentes, presentes na gestão em enfermagem contemporânea, tanto nos países desenvolvidos como no Brasil.

\section{Colaborações:}

1. concepção, projeto, análise e interpretação dos dados: Claudia Aparecida Dias, Dilma Costa Santos e Luiza Hiromi Tanaka;

2. redação do artigo e revisão crítica relevante do conteúdo intelectual: Luciana de Oliveira Matias, Maria Lucia Silva Servo e Carmem Lúcia Albuquerque de Santana;

3. aprovação final da versão a ser publicada: Claudia Aparecida Dias, Dilma Costa Santos e Luiza Hiromi Tanaka. 


\section{Referências}

1. Jesus Rocha MC, Sousa P, Martins M. A opinião dos enfermeiros diretores sobre a intervenção do enfermeiro chefe. Investig Enferm: Imagen Desarr [Internet]. 2016 [cited 2017 Aug 19];18(2):89-105. Available from: http://www.redalyc.org/articulo. oa?id=145245624007

2. Carvalho JFS, Chaves LDP. Supervisão de enfermagem no contexto hospitalar: uma revisão integrative. Rev Eletr Enf [Internet]. 2011 jul/set [cited 2016 Sep 8];13(3):546-53. Available from: http://www.fen.ufg.br/revista/v13/n3/v13n3a21. htm

3. Correia VS, Servo ML. Processo de supervisão social em enfermagem: possibilidade de transformação do modelo assistencial. Rev Saúde Com. 2013;9(3):90-102.

4. Presotto GV, Ferreira MBG, Contim D, Simões ALA. Dimensões do trabalho do enfermeiro no contexto hospitalar. Rev Rene. 2014 set-out; 5(5):760-70.

5. Lima AMV, Peduzzi M, Miyahara CTS, Fujimori E, Veríssimo MLR, Bertolozzi MR. Supervisão de trabalhadores de enfermagem em Unidade Básica de Saúde. Trab educ saúde (online). 2014 set-dez [cited 2017 Jan 24];12(3):57793. Available from: http://www.scielo.br/scielo. php?pid=S1981-77462014000300577\&script $=$ sci abstract

6. Romero JAV, Heredero CP. The strategic impact of clinical practice guidelines in nursing on the managerial function of supervision. Rev esc enferm USP. 2013 oct;47(5):1233-8.

7. Ning TJ, Costello J. Implementing clinical nursing supervision in Singapore hospitals. GSTF J Nurs Health Care [Internet]. 2017 Dec [cited 2018 Mar 7];5(1). Available from: file:///C:/Users/claud/ Downloads/1257-61-3222-1-10-20180126.pdf

8. Cutcliffe JR, Sloan G. Competences for Clinical Supervision in Psychiatric/Mental Health Nursing. In: Santos J, Cutcliffe J, editors. European Psychiatric/Mental Health Nursing in the 21st Century. Principles of Specialty Nursing (Under the auspices of the European Specialist Nurses Organisations (ESNO)). Australian College of Mental Health Nurses: Springer, Cham; 2018. p.123-39 [cited 2018 June 9]. Available from: https://link.springer.com/ chapter/10.1007/978-3-319-31772-4_10\#citeas
9. Townsend K, Wilkinson A, Kellner A. Opening the black box in nursing work and management practice: the role of ward managers. J Nurs Manag [Internet]. 2015 Mar [cited 2017 May 31];23:211-20. Avaliable from: http://onlinelibrary.wiley.com.proxyub. rug.nl/doi/10.1111/jonm.12115/epdf

10. Araújo DR, Sampaio FMC, Castro MC, Pinheiro SAV, Macedo AP. Teste no tempo: da teoria clássica da administração à organização atual do trabalho de Enfermagem. Rev Enf Ref. 2014 maio-jun;IV(2):111-20

11. Carvalho NA, Gama BMBM, Salimena AMO. A supervisão sob a ótica dos enfermeiros: reflexos na assistência e trabalho em equipe. Rev Adm Saúde [Internet]. 2017 out-dez [cited 2018 Apr 22];69(17). Available from: http://dx.doi.org/10.23973/ ras. 69.68

12. Moscovici S. Representações Sociais: investigações em psicologia social. 9a ed. Petrópolis: Vozes; 2012.

13. Sampire RH, Collado CF, Lúcio MDPB. Metodologia da pesquisa. Tradução Daisy de Moraes. 5a ed. Porto Alegre: Penso; 2013.

14. Brasil. Conselho Nacional de Saúde. Resolução n. 510, de 7 de abril de 2016. Dispõe sobre as normas aplicáveis a pesquisas em Ciências Humanas e Sociais. Brasília (DF); 2016 [cited 2018 Apr 30]. Available from: http://conselho.saude.gov.br/ resolucoes/2016/reso510.pdf

15. Weaver SH, Lindgren TG, Cadmus E, Flynn L, Thomas-Hawkins C. Report from the night shift how administrative supervisors achieve nurse and patient safety. Nurs Adm Q [Internet]. 2017 Oct/Dec [cited 2018 June 6];41(4):328-36. Available from: https://www.ncbi.nlm.nih.gov/pubmed/28859001

16. Correia VS, Servo MLS. Representações sociais das enfermeiras sobre supervisão: da tradicional à social. Rev Enferm UFPE (online). 2012 nov [cited 2016 Feb 17];6(11):2753-60. Available from: https:// periodicos.ufpe.br/revistas/revistaenfermagem/ article/view/7644

17. Chaves LDP, Mininel VA, Silva JAM, Alves LR, Silva MF, Camelo SHH. Supervisão de enfermagem para a integralidade do cuidado. Rev Bras Enferm [Internet]. 2017 set/out [cited 2018 May 23];70(5):1106-11. Available from: http://www.scielo.br/scielo.php?script=sci_ arttext $\&$ pid $=S 003471672017000501106 \& \operatorname{lng}=$ pt. http://dx.doi.org/10.1590/0034-7167-2016-0491 
18. Silva JS, Fortuna CM, Pereira MJB, Matumoto S, Santana FR, Marciano FM, et al. Supervision of Community Health Agents in the Family Health Strategy: the perspective of nurses. Rev esc enferm USP. 2014 oct;48(5):899-906.

19. Santos JLG, Pestana AL, Guerrero P, Meirelles BSH, Erdmann AL. Práticas de enfermeiros na gerência do cuidado em enfermagem e saúde: revisão integrativa. Rev bras enferm. 2013 abr;66(2):257-63.

20. Lanzoni1 GMM, Magalhães ALP, Costa VT, Erdmann AL, Andrade SR, Meirelles BHS. Tornando-se gerente de enfermagem na imbricada e complexa fronteira das dimensões assistencial e gerencial. Rev Eletr Enf. 2015 abr-jun;17(2):322-32.

21. Carvalho NA, Gama BMBM, Salimena AMO. A supervisão sob a ótica dos enfermeiros: reflexos na assistência e trabalho em equipe. Rev Adm Saúde [Internet]. 2017 out/dez [cited 2018 May 26];17(69). Available from: http://dx.doi.org/10.23973/ras.69.68

22. Senna MH, Drago LC, Kirchner AR, Santos JLG, Erdmann AL, Andrade SR. Significados da gerência do cuidado construídos ao longo da formação profissional do enfermeiro. Rev Rene. 2014 mar-abr;15(2):196-205.

23. Santos JLG, Prochnow AG, Silva DC, Silva RM, Leite JL, Erdmann AL. Prazer e sofrimento no exercício gerencial do enfermeiro no contexto hospitalar. Esc Anna Nery. 2013 mar;17(1):97-103.

24. Gunawan J, Aungsuroch Y, Fisher ML. Factors contributing to managerial competence of first linenurse managers: a systematic review. Int J Nurs Pract [Internet]. 2018 [cited 2018 June 16];24:e12611. Available from: https://onlinelibrary.wiley.com/ doi/epdf/10.1111/ijn.12611

25. Tironi NM, Bernardino E, Haddad MCL, Nimtz MA, Torres DG, Peres AM. Assignments and competencies of nursing managers: a descriptive exploratory research. Online braz j nurs [Internet]. 2017 Mar [cited 2018 June 16];16(1):130-9. Available from: http://www.objnursing.uff.br/index.php/ nursing/article/view/5601

Recebido: 19 de julho de 2018 Aprovado: 23 de outubro de 2018 Publicado: 26 de dezembro de 2018

A Revista Baiana de Enfermagem utiliza a Licença Creative Commons - Atribuição-NãoComercial 4.0 Internacional. https://creativecommons.org/licenses/by-nc/4.0/

Este artigo é de acesso aberto distribuído sob os termos da Licença Creative Commons (CC BY-NC). Esta licença permite que outros remixem, adaptem e criem a partir do seu trabalho para fins não comerciais. Embora os novos trabalhos tenham de lhe atribuir o devido crédito e não possam ser usados para fins comerciais, os usuários não têm de licenciar esses trabalhos derivados sob os mesmos termos. 